\title{
Sensitivity of the Developing Rat Brain to Hypobaric/Ischemic Damage Parallels Sensitivity to $\mathbf{N}$-Methyl-Aspartate Neurotoxicity
}

\author{
C. Ikonomidou, J. L. Mosinger, K. Shahid Salles, J. Labruyere, and J. W. Olney \\ Department of Psychiatry, Washington University School of Medicine, St. Louis, Missouri 63110
}

The endogenous excitotoxin, glutamate (Glu), acting at the N-methyl-aspartate (NMA) subtype of Glu receptor, is thought to play a major role in hypoxic/ischemic neuronal degeneration. In the present study, the sensitivities of the developing rat CNS to hypoxic/ischemic neuronal degeneration and to the neurotoxic action of NMA were compared at various postnatal ages. In the hypoxic/ischemic experiments, ischemia was produced by unilateral common carotid artery ligation and hypoxia by subjecting the pups to a partial vacuum. Keeping the duration of the hypobaric episode constant at $75 \mathrm{~min}$ for all age groups, we observed that the vulnerability of the immature brain to hypobaric/ischemic damage increased during the early neonatal period (days 2-4), reached a peak at day 6 and then diminished progressively with increasing age. In the second part of the study, NMA was microinjected unilaterally into the head of the caudate nucleus at various postnatal ages (2-80 d). In the early neonatal period (days 2-6), injections of relatively small doses of NMA (6-15 $\mathrm{nmol}$ ) produced a dose-dependent widespread excitotoxic reaction throughout the forebrain with peak sensitivity being observed on day 6 . The cytotoxic reactlon to NMA was identical In appearance and time course to that induced by hypobaric/ischemic methods. With increasing age, the excitotoxic response to a given dose of NMA decreased progressively and the lesions became more strictly confined to the injection site. Cell populations most sensitive to NMA toxicity in the 2-10 d period closely correlated with those most vulnerable to hypoxia/ischemia, and sensitivity to both types of injury reached a peak at $6 \mathrm{~d}$. These findings reinforce other evidence linking an excitotoxic mechanism and the NMA subtype of Glu receptor to hypoxic/ischemic brain damage and suggest that there may be a period during development when NMA receptors are hypersensitive to excitotoxic stimulation, thus rendering the neurons possessing such receptors hypervulnerable to hypoxic/ischemic damage.

The endogenous excitatory amino acids (EAA), glutamate (Glu) and aspartate, are leading neurotransmitter candidates and also neurotoxins (excitotoxins) capable of destroying CNS neurons by excessive activation of EAA receptors. It is believed that an excitotoxic mechanism may underly hypoxic/ischemic neuronal

\footnotetext{
Received Dec. 8, 1988; revised Jan. 31, 1989; accepted Feb. 1, 1989.

Supported in part by NIMH Research Scientist Award MH 38894 (J.W.O.) and HHS grants HD 24237 and ES 07066.

Correspondence should be addressed to John W. Olney, M.D., Washington University School of Medicine, Department of Psychiatry, 4940 Audubon Avenue, St. Louis, MO 63110.

Copyright (C) 1989 Society for Neuroscience $0270-6474 / 89 / 082809-10 \$ 02.00 / 0$
}

degeneration in conditions such as stroke, cardiac arrest, and perinatal asphyxia. This hypothesis is supported by evidence that Glu and aspartate accumulate extracellularly in the CNS under hypoxic/ischemic conditions (Benveniste et al., 1984; Hagberg et al., 1987) and that agents with known EAA antagonist properties protect against hypoxic/ischemic neuronal degeneration both in vitro (Rothman, 1984; Weiss et al., 1986; Clark and Rothman, 1987; Goldbcrg et al., 1987; Olncy ct al., 1988b) and in vivo (Simon et al., 1984; Gill et al., 1987; Lawrence et al., 1987; McDonald et al., 1987; Park et al., 1987; Boast et al., 1988; Kochhar et al., 1988; Olney et al., 1988a, 1989; Prince and Freeser, 1988; Steinberg et al., 1988). Several EAA receptor subtypes have been identified (Watkins and Olverman, 1987), each being named by a potent EAA agonist [ $N$ methyl aspartic acid (NMA), kainic acid, quisqualic acid] to which it is differentially sensitive. The relative importance of each receptor subtype in the mediation of hypoxic/ischemic brain damage remains to be determined. Recently, we described a new model for studying hypoxic/ischemic neuronal degeneration in infant rat brain (Olney et al., 1987; Ikonomidou et al., 1988, 1989). Hypoxic/ischemic neuronal degeneration is induced in this model by unilateral carotid artery ligation followed by exposure to a partial vacuum. The cytopathological reaction induced in infant rat brain by this hypobaric/ischemic approach is indistinguishable from that induced in the brains of infant rats and monkeys by systemic administration of Glu (Olney et al., 1987; Ikonomidou et al., 1988, 1989). Pretreatment with MK-801, a potent and selective NMA antagonist, powerfully protects the infant rat brain against hypobaric/ischemic damage (Olney et al., 1988, 1989).

The recent observation by McDonald et al. (1988) that the immature rat brain is quite sensitive to the neurotoxic action of NMA is of considerable interest in that kainic acid, which is very powerful in destroying neurons in adult rat brain, induces very little neurotoxic reaction when injected into neonatal rat brain (Campochiaro and Coyle, 1978; Wolf and Keilhoff, 1984). Apparently, at this stage of development, kainic acid receptors are not mature enough to mediate an excitotoxic reaction. Inherent in these findings is the important implication that, in certain stages of development, hypersensitivity of NMA receptors combined with hyposensitivity of non-NMA receptors might result in preferential involvement of NMA receptors in the mediation of hypoxic/ischemic brain damage; efficacy of NMA antagonists in preventing such brain damage lends support to this interpretation (McDonald et al., 1987; Olney et al., 1988a, 1989; Prince and Freeser, 1988). To further explore the role of NMA receptors in perinatal hypoxic/ischemic brain damage, we have compared the response of the developing CNS to the direct intracerebral injection of NMA with its response to hypobaric/ ischemic conditions at various stages of development. 


\section{Materials and Methods}

Sprague-Dawley rats at various postnatal ages $(2,4,10,14,20,30$, and $80 \mathrm{~d}$ ), which were bred and housed in our laboratories under environmentally controlled conditions, were used in these experiments. Pups were housed together with their mothers in individual cages until the age chosen for study or until weaning on day 24 (the day of birth was considered as day 0). In the first series of experiments, unilateral ligation of the common carotid artery was performed under halothane anesthesia. After recovery from anesthesia, the animals were placed in a hypobaric chamber (air volume, $2370 \mathrm{ml}$ ), which was immersed in a water bath maintaining the interior of the chamber at $36 \pm 0.5^{\circ} \mathrm{C}$. The number of animals in the chamber varied among different age groups and was chosen to assure that the total body mass per chamber was maintained constant in all experiments $(180-210 \mathrm{gm})$. Underlying this precaution is the assumption that there is a relatively stable correlation between an animal's body mass and its oxygen consumption. The pressure in the chamber was lowered gradually over a $1 \mathrm{~min}$ period to $225 \mathrm{~mm} \mathrm{Hg}$ and maintained at this level for $75-120 \mathrm{~min}$, then raised to $760 \mathrm{~mm}$ $\mathrm{Hg}$ over a $1 \mathrm{~min}$ period. Two hours after hypobaric exposure all animals were deeply anesthetized and killed by perfusion fixation for histopathological examination of their brains.

In the second series of experiments, animals received a microinjection of NMA (Sigma, St. Louis, MO; 6-15 nmol) or saline into the head of the caudate nucleus. The rats were anesthetized with halothane and were placed in a Kopf stereotaxic frame. For pups 2-10 d of age, the head was fixed between 2 acrylic blocks that were previously molded to fit the lateral shape of the skull. The neck and jaw rested on a subaxic guide bar. The coordinates for stereotaxic injections into the head of the caudate nucleus of 2-and 6-d-old rats were determined empirically. For older rats, the injections were performed according to established age-appropriate stereotaxic guidelines (Koenig and Klippel, 1967; Sherwood and Timiras, 1970). A Hamilton syringe mounted with a 30 gauge needle was used for injection of saline $(0.2 \mu \mathrm{l})$ or NMA $(6-15 \mathrm{nmol} / 0.2$ $\mu l)$. NMA was dissolved in distilled water and the $\mathrm{pH}$ adjusted to a physiological range (7.3-7.4) by addition of $\mathrm{NaOH}$. All microinjections were performed over a 2 min period, and the needle remained in the brain for $2 \mathrm{~min}$ thereafter. All animals were allowed to survive $4 \mathrm{hr}$, then were deeply anesthetized with an overdose of pentobarbital and perfused through the heart and ascending aorta with a solution of paraformaldehyde $(1 \%)$ and glutaraldehyde $(1.5 \%)$ in phosphate buffer. After perfusion for $15 \mathrm{~min}$ the brains were removed and processed for light and electron microscopy as previously described (Olney, 1971).

Transverse sections, $1 \mu \mathrm{m}$ thick, were cut at 2 levels $[2.3$ and $5.6 \mathrm{~mm}$ anterior to the zero point at the age of $10 \mathrm{~d}, 3.2$ and $6.5 \mathrm{~mm}$ anterior to the zero point at the age of $20 \mathrm{~d}, 4.1$ and $7.5 \mathrm{~mm}$ anterior to the zero point at the age of $30 \mathrm{~d}$, according to the atlas of Sherwood and Timiras (1970) and 7.47 and $3.99 \mathrm{~mm}$ at the age of $80 \mathrm{~d}$, according to the atlas of Koenig and Klippel (1967)], which permitted visualizing brain regions vulnerable to both hypobaric/ischemic damage and the neurotoxic action of NMA. For purposes of quantifying the damage in a given region, the number of acutely necrotic neurons (NN) were counted on a single section containing that region, then the counts for the several regions were summed to give a total NN score for each brain. Counts were limited to "bull's-eye" profiles, i.e., neurons exhibiting vacuous swollen perikarya and pyknotic changes (chromatin clumping) in the nucleus. One-way analyses of variance (ANOVAs) were used to analyze the NN data, and subsequent pairwise comparisons were conducted following significant $F$ ratios in the 1-way analyses (Winer, 1971).

\section{Results}

Hypobaric/ischemic brain damage

Consistent with previous observations (Olney et al., 1987; Ikonomidou et al., 1988, 1989), at $10 \mathrm{~d}$ of age, hypobaric/ischemic conditions caused a pattern of brain damage ipsilateral to the carotid ligation affecting the medial habenulum, caudate nucleus (Fig. 1a), septum (Fig. 2a), frontoparietal ncocortices (Fig. 3a), hippocampus, olfactory tubercle, and several thalamic nuclei. The cytopathological changes consisted predominantly of edematous swelling of dendritic processes and acute edematous degeneration of neuronal cell bodies with pyknotic changes occurring early in the nucleus (Figs. 1-3). Some neurons manifested a less acute process of vacuolar condensation, which may represent the delayed neurodegenerative response to transient Glu exposure that has been described in cortical (Choi et al., 1987) and hippocampal (Rothman et al., 1987) cell cultures. An evaluation of delayed neurodegenerative changes was not included in the scope of the present study.

In general, the acute response of the rat brain to hypobaric/ ischemic conditions at different stages of development revealed age-related differences in the incidence, severity, and pattern of neurodegenerative changes. When all age groups were exposed to exactly the same conditions $(225 \mathrm{~mm} \mathrm{Hg}$ for $75 \mathrm{~min})$, the severity of brain damage increased in the interval from days 2 to 6 and then progressively decreased at subsequent ages with no acute brain damage being evident in 80-d-old animals (Fig. $5 A$ ). There was a large inequality of within-group variances among the different age groups. Much of this disparity was due to high frequencies of zero NN scores in the groups killed at 2 and $80 \mathrm{~d}$. To compensate for this heterogeneity of variance, a logarithmic transformation $[\log (x+1.5)]$ was performed on the raw data (NN scores). An ANOVA of these transformed data confirmed that the groups differed significantly in amounts of hypobaric/ischemic brain damage, $F(7,133)=18.36, p<0.0009$. Subscquent pairwise comparisons indicated that a significantly greater amount of brain damage was found at $6 \mathrm{~d}$ compared with $2 \mathrm{~d}, F(1,133)=93.61, p<0.0009,4 \mathrm{~d}, F(1,133)=14.76$, $p<0.0009,10 \mathrm{~d}, F(1,133)=9.68, p<0.002$, or compared with the avcrage amount of damage incurred across $14-80 \mathrm{~d}$, $F(1,133)=58.57, p<0.0009$.

It is noteworthy that in the very early nconatal period (day 2), rat pups were quite resistant to hypobaric/ischemic brain damage under these protocol conditions. When the hypobaric exposure time was extended to $120 \mathrm{~min}$, pups at this age did sustain brain damage, but the severity of such damage was less than that observed in 4- to 10-d-old pups exposed to hypobaric conditions for only $75 \mathrm{~min}$ (Fig. $5, A, B$ ). The survival rate of 2 -d-old rats after $75 \mathrm{~min}$ of hypobaric exposure was $100 \%$ and after $120 \mathrm{~min}$ exposure was $85 \%$, which is comparable to the survival rate of $6-$ and 10 -d-old animals (90 and $88 \%$, respectively) after an exposure time of $75 \mathrm{~min}$. Similarly, when the hypobaric cxposure period for 4-d-old pups was extended from 75 to $90 \mathrm{~min}$, there was an increase in brain damage scores (Figs. $5, A, B$ ) and decrease in survival rate (from 100 to $85 \%$ ).

Of particular interest was the observation that hypobaric/ ischemic conditions in the younger age groups (2-6 d) were associated with a pronounced hemorrhagic diathesis. A subarachnoid hematoma over the cerebellum was consistently observed and hemorrhagic foci were also characteristically distributed diffusely throughout the forebrain, with most such hemorrhages being in the close proximity of an acute neuronal degenerative focus.

Different brain regions showed different times of peak sensitivity to hypobaric/ischemic damage (Table 1). In the youngest age groups (days 2-4), acute neuronal lesions were found predominantly in the neocortex and caudate nucleus with the hippocampus, thalamus, and olfactory tubercle being relatively resistant. At $6 \mathrm{~d}$, a more generalized reaction frequently involving many neuronal groups was observed (Table 1). The frontoparietal neocortices were frequently damaged throughout days 4 10 but became totally resistant by day 14 . At 10 and $14 \mathrm{~d}$ of age, ncurons in periventricular regions (medial habenulum, head and tail of the caudate nucleus, mediodorsal thalamus) were the most sensitive to hypobaric/ischemic degeneration (Table 1). 

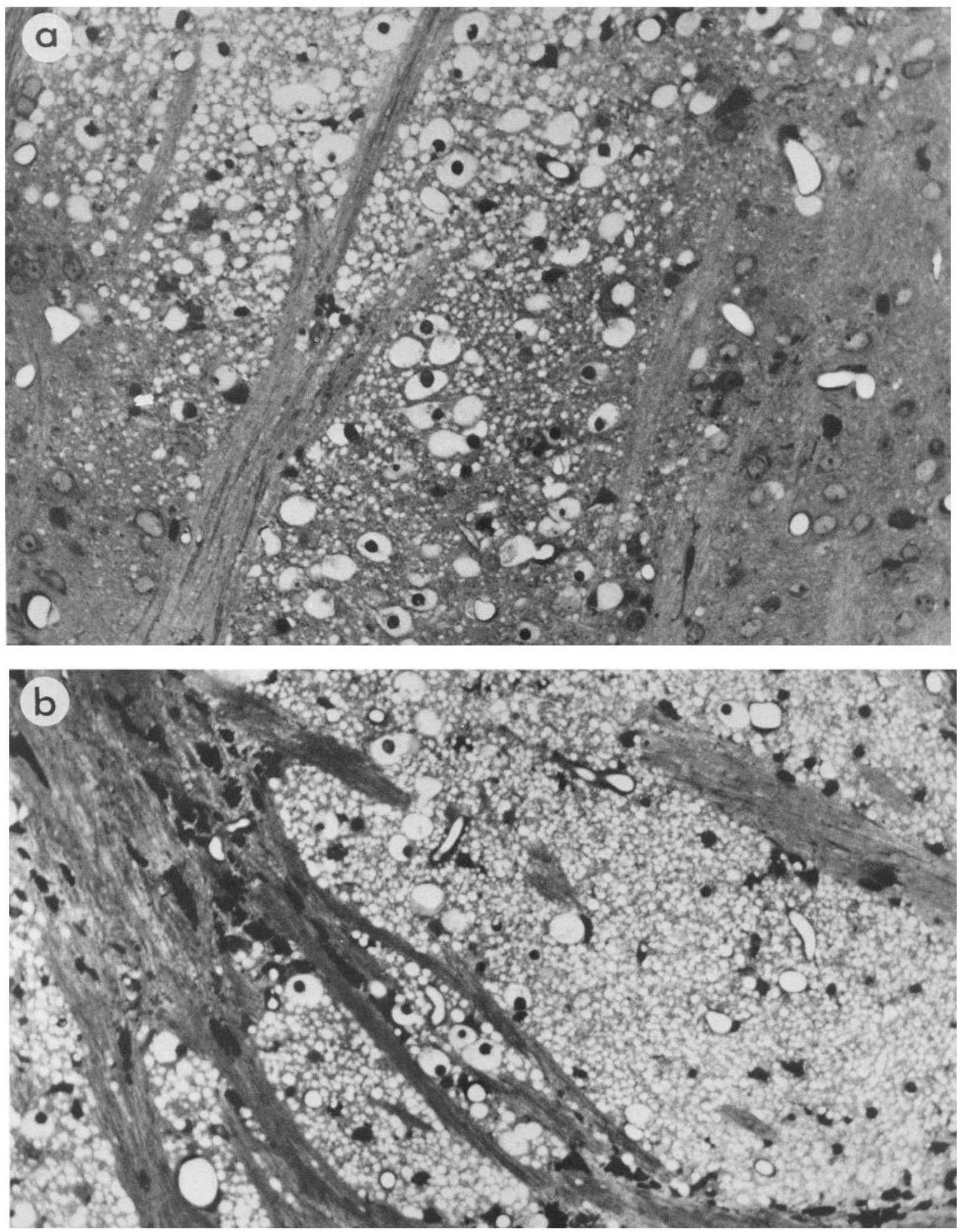

Figure 1. a. An acute hypoxic/ischemic lesion in the head of the caudate nucleus of a 10-d-old rat caused by unilateral ligation of the common carotid artery and subsequent exposure of the rat pup to $225 \mathrm{~mm} \mathrm{Hg}$ for $75 \mathrm{~min}$. $b$. Head of the caudate nucleus of a 10-d-old rat that received an intrastriatal injection of $9 \mathrm{nmol} \mathrm{NMA} 4 \mathrm{hr}$ previously. Both lesions exhibit the features of excitotoxic injury characterized by profound dendritic swelling and degenerating neurons with swollen cytoplasm and dark pyknotic nuclei. $\times 260$. 

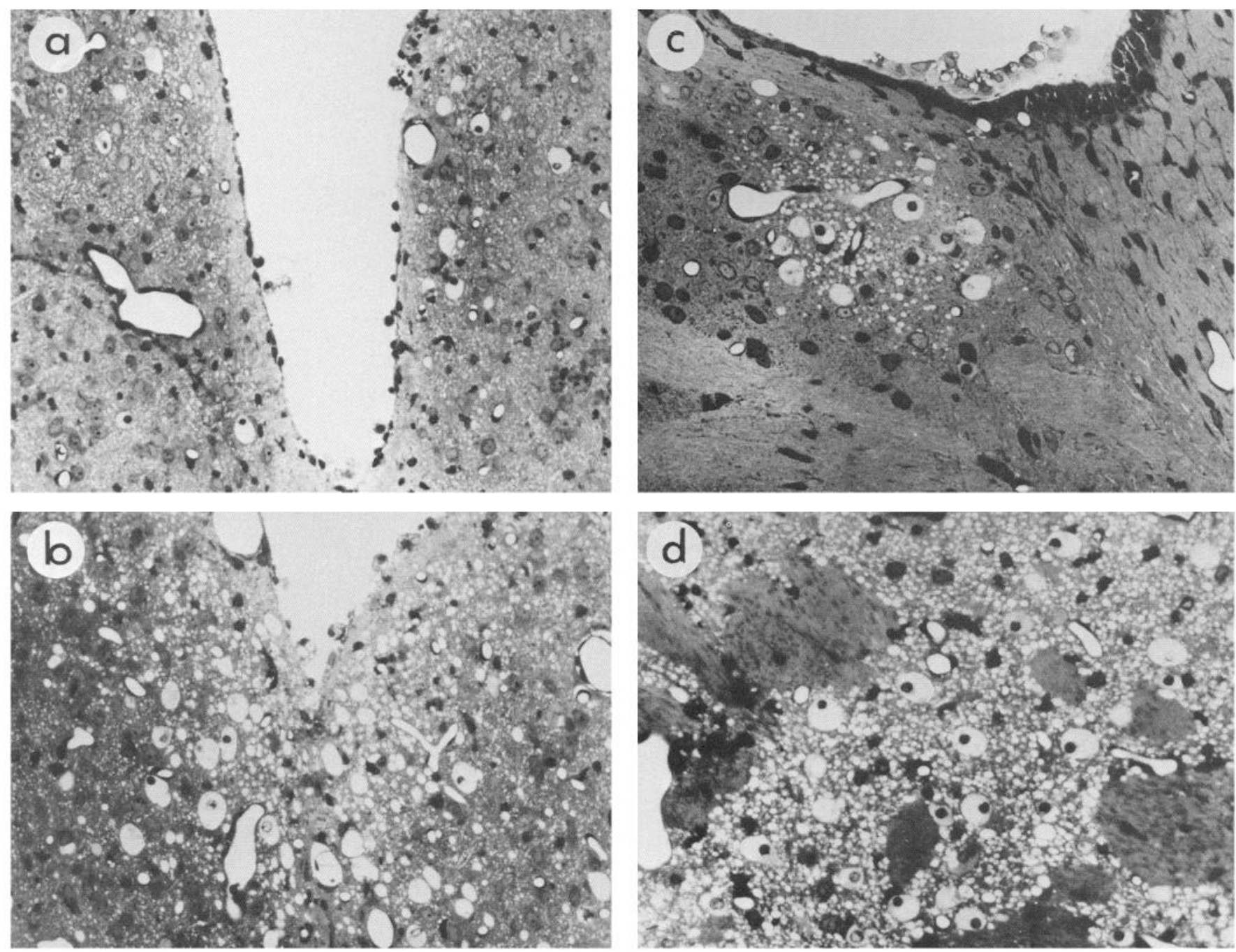

Figure 2. Panels at left illustrate medial septal lesions in 6-d-old rats caused by either hypoxia/ischemia $(a)$ or intrastriatal injection of 9 nmol NMA $(b)$. Panels at right depict hypoxic/ischemic $(c)$ and NMA-induced $(d)$ neuronal lesions in the tail of the caudate nucleus of a 10-d-old $(c)$ and a 6-d-old $(d)$ pup. $\times 200$.

Periventricular neurons adjacent to germinal matrix layers in the head and tail of the caudate nucleus were consistently vulnerable to hypoxic/ischemic degeneration at all ages between 4 and $14 \mathrm{~d}$.

\section{Microinjections of NMA}

In the early neonatal period (days 2-6), unilateral microinjection of NMA (6-15 nmol) produced a severe and widespread excitotoxic reaction throughout the forebrain, which in most cases involved neuronal groups both ipsi- and contralateral to the injection site. In this early period, but not later, a disseminated pattern of petechial hemorrhaging was observed as an additional response to the intrastriatal injection of NMA. The excitotoxic reaction was characterized by profound dendritic swelling, acute edematous degeneration of neuronal cell bodies, and pyknotic changes in the neuronal nuclei (Figs. 1-4). Neuronal groups that appeared particularly sensitive to the acute excitotoxic action of NMA included those located periventricularly in the head (Figs. 1b, 4) and tail (Fig. 2d) of the caudate nucleus, those in the medial (Fig. 2b) and lateral septum, the cingulate, frontal, and parietal neocortices (Fig. 3b), thalamus, and hippocampus. Immature cells comprising the periventricular germinal matrix rarely degenerated following NMA injection, but neurons adjacent to the germinal matrix were extremely vulnerable to degeneration (Fig. 4). Typically, in any given brain, these cells displayed advanced karyopyknotic changes in contrast to similar but less advanced changes noted in other striatal neurons. At older ages ( $>20 \mathrm{~d}$ ), microinjection of NMA into the head of the caudate nucleus produced only small lesions confined to the injection site.

In order to study possible age-related changes in the vulnerability of the developing brain to NMA neurotoxicity, we injected a fixed dose $(9 \mathrm{nmol})$ into the rat caudate nucleus at $2(n$ $=13), 6(n=14), 10(n=7), 20(n=8), 30(n=6)$, and $80(n$ $=5$ ) days of age and compared the severity of brain damage at various ages. In the $2-4 \mathrm{~d}$ period, the acute reaction was relatively pronounced, but it consisted of a more extensive display of dendritic swelling with less frequent necrosis of neuronal cell bodies than occurred on day 6 . The severity of acute brain damage appeared to be highest on day 6 (Fig. 6) and to decline 

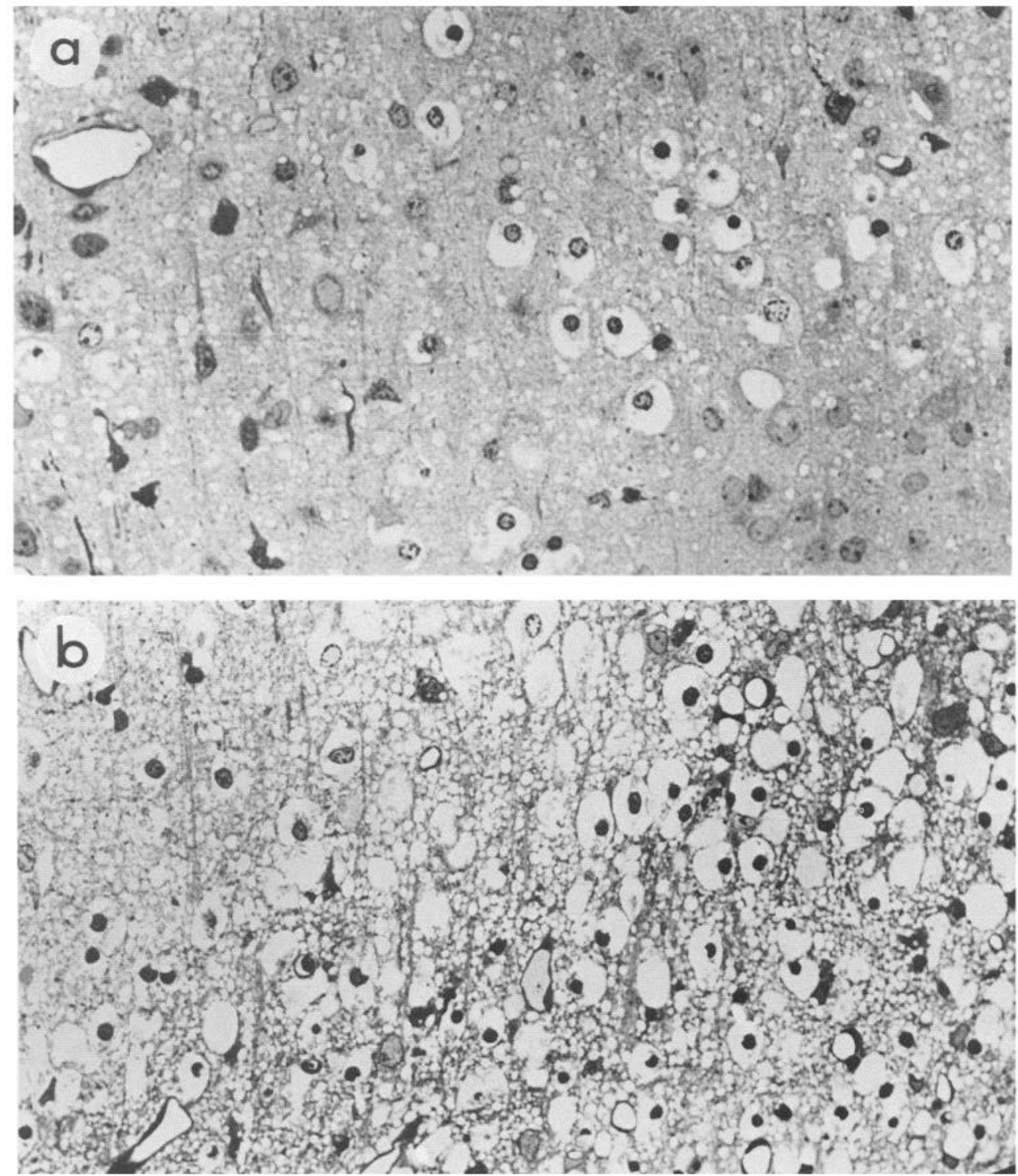

Figure 3. a, Acutely degenerating neurons in the parietal neocortex of a 10 -d-old rat $2 \mathrm{hr}$ following an episode of hypoxia/ischemia. $b$, Acutely degenerating neurons in the parietal neocortex of a 6-d-old rat following intrastriatal microinjection of 9 nmol NMA into the rostral part of the caudate nucleus. $\times 260$. progressively with age. On the 10th postnatal day, damage was limited to the caudate injection site and the superjacent frontal cortex. At $80 \mathrm{~d}$ of age, 9 nmol NMA produced small lesions in the immediate vicinity of the injection. Intrastriatal injection of saline did not produce lesions in any of the animals tested. ANOVA was performed on a logarithmic transformation of the NN scores (required due to a large inequality of within-group variances among the postnatal day groups), and this confirmed that the age groups differed significantly in severity of brain damage, $F(5,47)=77.38, p<0.0009$. A significantly greater amount of brain damage was found at $6 \mathrm{~d}$ compared with $2 \mathrm{~d}$, $F(1,47)=6.28, p<0.016,10 \mathrm{~d}, F(1,47)=80.44, p<0.0009$, and compared with the average amount of damage found at 20 $80 \mathrm{~d}, F(1,47)=305.15, p<0.0009$.

\section{Comparison of the sensitivity of different neuronal populations to hypoxia/ischemia and NMA}

As described above, the developing brain proved to be most sensitive to both hypoxia/ischemia and the neurotoxic action of NMA on the 6 th postnatal day. In order to determine whether
Table 1. Frequency ${ }^{a}$ and regional distribution of hypoxic/ischemic lesions at different ages

\begin{tabular}{lrlrrr} 
Region & $4 \mathrm{~d}$ & $6 \mathrm{~d}$ & $10 \mathrm{~d}$ & $14 \mathrm{~d}$ & $20 \mathrm{~d}$ \\
\hline Habenular nucleus & 33 & 70 & 75 & 79 & 0 \\
Caudate nucleus, rostral & 83 & 65 & 45 & 36 & 10 \\
Caudate nucleus, caudal & 92 & 75 & 80 & 71 & 80 \\
Thalamus, mediodorsal & 0 & 55 & 45 & 21 & 0 \\
Thalamus, lateral dorsal & 8 & 60 & 45 & 7 & 0 \\
Thalamus, ventral & 0 & 45 & 0 & 0 & 10 \\
Hippocampus & 0 & 20 & 0 & 0 & 0 \\
Subiculum, dorsal & 0 & 40 & 5 & 0 & 0 \\
Neocortex, frontal & 83 & 90 & 30 & 0 & 0 \\
Neocortex, parietal & 83 & 95 & 40 & 0 & 0 \\
Olfactory tubercle & 0 & 53 & 45 & 29 & 0 \\
Septum & 17 & 44 & 35 & 0 & 0
\end{tabular}

\footnotetext{
${ }^{a}$ Percentage of animals at a given age that had acutely necrotic neurons in a given
} region. 
Figure 4. Low-power view of the periventricular portion of the rostral part of the caudate nucleus in a 6-d-old rat that received intrastriatal microinjection of 9 nmol NMA 4 hr previously. The periventricular germinative matrix layer is intact. Note the acutely degenerating striatal neurons adjacent to the periventricular matrix. $\times 60$.

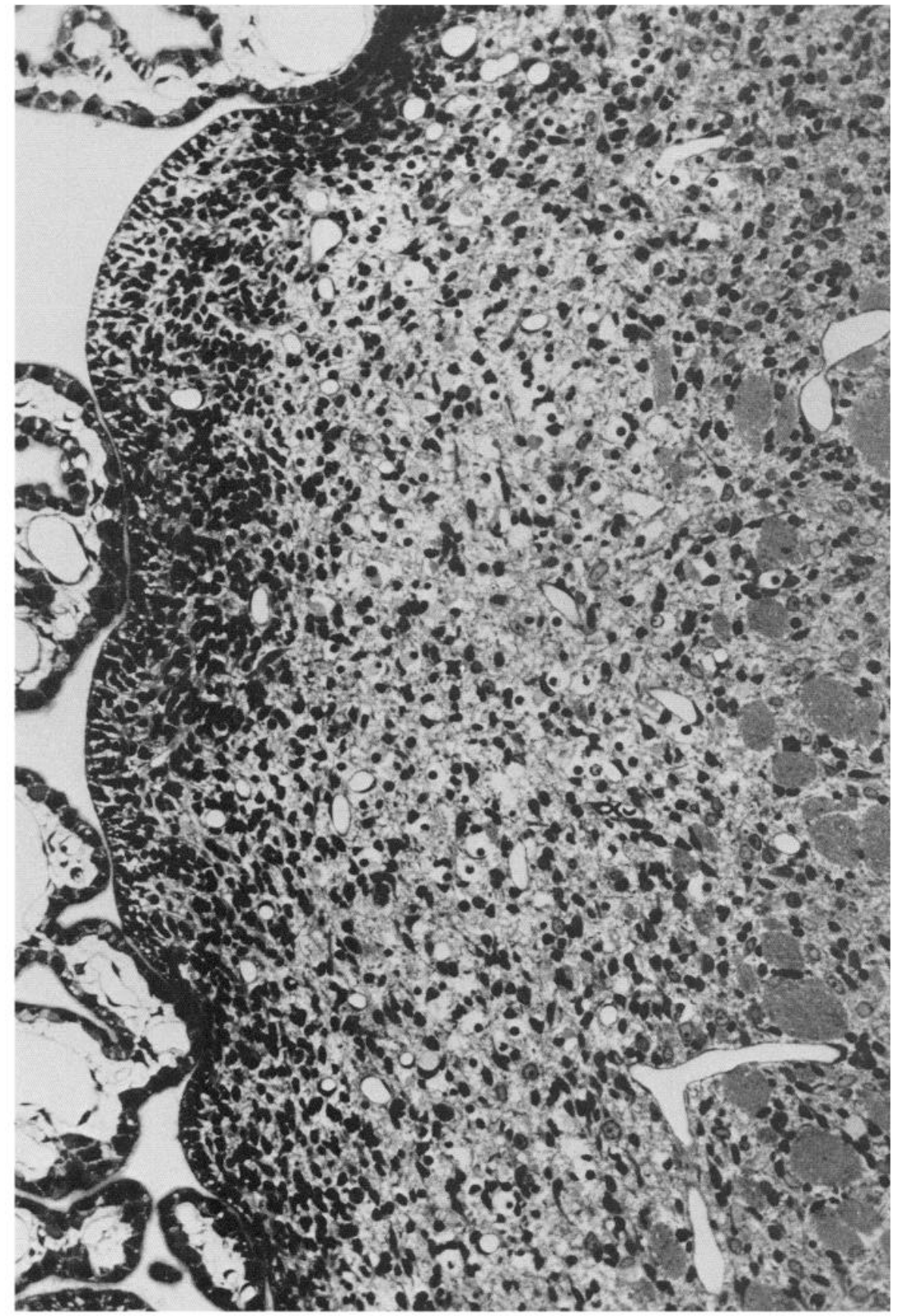

there is any correlation between the sensitivity of specific neuronal populations to NMA and their degenerative response to hypoxia/ischemia, we compared the severity of damage sustained by various neuronal groups under each condition on the 6 th postnatal day. Following unilateral microinjection of $9 \mathrm{nmol}$ NMA into the caudate nucleus of 6-d-old rats, the most severely damaged brain regions, as determined by NN counts, were (in order of decreasing severity): parietal neocortex, frontal neocortex, caudate nucleus, septum, hippocampus, and thalamus (Table 2). Particularly striking was the observation that these brain regions were selectively damaged bilaterally even though NMA was injected only unilaterally. The damage was not as severe on the side contralateral to the injection, but the neuronal groups damaged most severely on the contralateral side were the same as those damaged most severely on the ipsilateral side.

As Table 1 demonstrates, in 6-d-old rats subjected to hypoxic/ ischemia the frontal and parietal neocortices and the caudate nucleus were regions very frequently damaged and the septum, hippocampus, thalamus, and olfactory tubercle were damaged with moderate frequency. Therefore, there is a strong positive correlation between the sensitivity of specific neuronal groups to NMA toxicity and hypoxic/ischemic degeneration on the 6th postnatal day. There were 2 minor exceptions: (1) The septum was not quite as severely damaged by hypoxia/ischemia as by NMA, probably due to its midline location providing it with adequate vascular perfusion from the brain side contralateral 

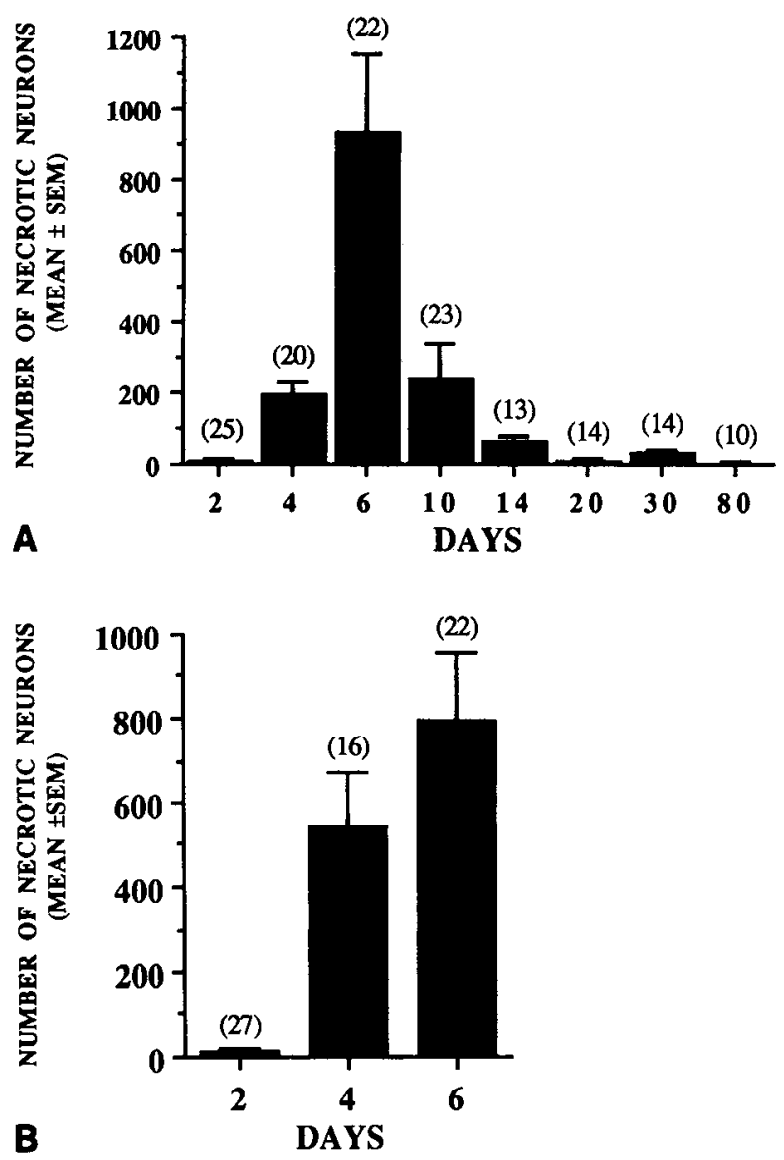

Figure 5. A, Mean scores $( \pm$ SEM) for hypoxic/ischemic brain damage in different age groups (for description of the rating system see Materials and Mcthods). All animals wcre subjected to exactly the same conditions $(225 \mathrm{~mm} \mathrm{Hg} \times 75 \mathrm{~min}$ ). Pups at the age of $6 \mathrm{~d}$ show the highest number of necrotic neurons. Pups aged $2 \mathrm{~d}$ are resistant to hypoxic/ischemic neuronal injury under these protocol conditions. $B$, Mean number of necrotic neurons ( \pm SEM) in the brains of 2-, 4-, and 6-d-old rats after the exposure time to hypobaric conditions had been adjusted in order to achieve a mortality rate of $10-15 \%$. The exposure times were 120 min for 2-d-old, $90 \mathrm{~min}$ for 4-d-old, and $75 \mathrm{~min}$ for 6-d-old pups. Under these protocol conditions there is an increase of scores for acute neuronal injury in both 2- and 4-d-old animals, but animals at $6 \mathrm{~d}$ of age still display the highest susceptibility to hypoxia/ischemia. The numbers at the top of each column represent the number of animals used in each age group.

to the carotid ligation. (2) Medial habenular neurons were more frequently and more severely damaged by hypoxia/ischemia. Medial habenular damage did occur in $40 \%$ of the NMA-injected brains, but it was not very pronounced, probably because these neurons were at a considerable distance from the injection site.

\section{Discussion}

Here we show that vulnerability of the rat CNS to hypoxic/ ischemic neuronal degeneration changes during development. When rats of different postnatal ages are exposed to the same hypoxic/ischemic conditions, those aged 4-14 d suffer the most severe acute hypoxic/ischemic neuronal injury, with peak severity occurring at $6 \mathrm{~d}$ of age. Newborn rats and animals older than $20 \mathrm{~d}$ are relatively less sensitive to hypoxic/ischemic damage. In addition, we show (1) that injection of nanomolar amounts of the potent Glu analog, NMA, into the infant rat brain causes

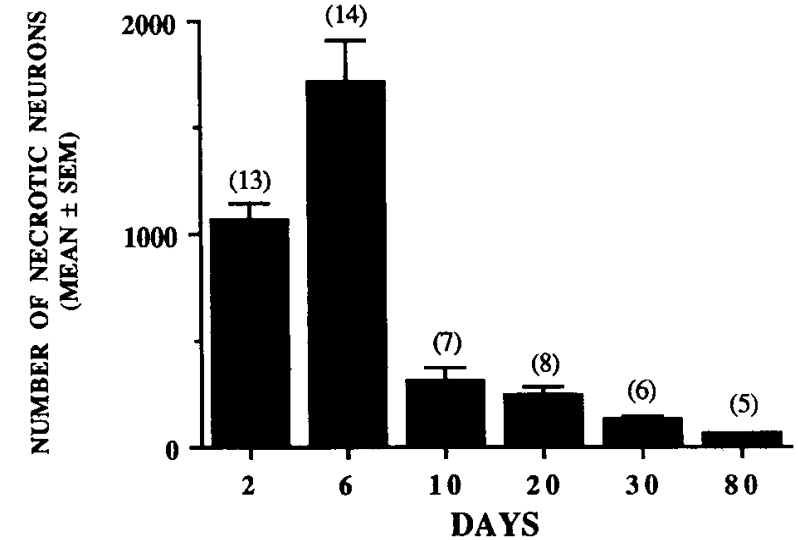

Figure 6. Mean number of neurons $( \pm \mathrm{SEM})$ destroyed in rat brain at various ages by intrastriatal microinjection of $9 \mathrm{nmol}$ NMA (for description of the rating system, see Materials and Methods). Pups aged $6 \mathrm{~d}$ show the highest susceptibility to the neurotoxic effect of NMA. Note that this age group proved to be most susceptible to hypoxic/ ischemic neuronal injury as well (Fig. 5, $A, B$ ). The numbers on top of each column represent the number of animals used in each age group.

cytopathological changes that appear identical to those associated with hypoxia/ischemia, (2) that sensitivity of the immature rat brain to NMA toxicity varies with developmental age, the period between postnatal days 4 and 14 being a period of relative hypersensitivity, and (3) that the infant rat brain is most sensitive to NMA toxicity on the 6 th postnatal day. The latter observation correlates well with the recent demonstration by McDonald et al. (1988) that NMA is much more potent as a neurotoxin when injected into rat brain on the 7 th postnatal day than when injected into adult rat brain. Our observation that the sensitivity of the immature rat brain to hypoxic/ischemic damage undergoes changes during development that closely parallel changes in sensitivity to the excitotoxic action of NMA supports the hypothesis that hypoxic/ischemic damage in infant rat brain is caused by an endogenous excitotoxin acting at the NMA subtype of Glu receptor. This interpretation is further reinforced by the identical histological appearance of neurons undergoing acute degeneration following exposure to Glu, NMA, or hypoxia/ischemia (Ikonomidou et al., 1988, 1989), and by evidence that MK-801, a powerful and selective antagonist of both the excitatory (Wong et al., 1986) and neurotoxic (Olney et al., 1987) actions of NMA, also powerfully protects the infant rat brain from hypoxic/ischemic injury (McDonald et al., 1987; Olney et al., 1988a, 1989).

Our findings are consistent with the proposal that there is a

Table 2. Severity and regional distribution of NMA-induced brain damage in 6-d-old rats

\begin{tabular}{llc} 
& \multicolumn{2}{c}{ Mcan nccrotic ncuron counts } \\
\cline { 2 - 3 } Brain regions & Ipsilateral & Contralateral \\
\hline Parietal cortex & 325 & 184 \\
Frontal cortex & 277 & 81 \\
Caudate nucleus & 191 & 90 \\
Septum & 153 & 119 \\
Hippocampus & 58 & 56 \\
Thalamus & 52 & 47 \\
\hline
\end{tabular}


period during ontogenesis when NMA receptors in the immature rat brain are hypersensitive to excitatory stimulation and that this causes neurons possessing such receptors to be hypervulnerable to hypoxic/ischemic degeneration. Other possible explanations for the extreme sensitivity of infant rat brain to hypoxic/ischemic damage might be considered. For example, since impairment of energy-dependent uptake systems for Glu and aspartate is thought to play a major role in the extracellular accumulation of these endogenous excitotoxins during hypoxia/ ischemia (Benveniste et al., 1984; Drejer et al., 1985; Silverstein et al., 1986), a reduced capacity (due to immaturity) of such uptake systems might be posited as an explanation for the hypervulnerability of infant brain to hypoxic/ischemic damage. Alternatively, or additionally, it is possible that immaturity of the circulatory system and high sensitivity of vascular autoregulatory mechanisms to oxygen deprivation might make the infant brain subject to a more pronounced vascular perfusion deficit resulting in greater energy impairment. However, the observation that the rat brain is relatively resistant to hypoxic/ ischemic injury on postnatal day 2 compared with day 6 or 10 would argue against these explanations. Gradual maturation of uptake systems for Glu and increasing vascularity might partially account for the decreasing sensitivity of the rat brain at ages beyond the 6 th postnatal day, but they would not explain the observed increasing sensitivity of the developing brain to oxygen deprivation during the first postnatal week.

It is of interest that the infant rat brain, although most sensitive to both NMA and hypoxia/ischemia on the 6th postnatal day, was relatively sensitive to NMA and resistant to hypoxic/ ischemic degeneration on day 2 . This discrepancy might be explained in terms of the dependence of Glu uptake systems on energy metabolism and a greater resistance of metabolic systems to hypoxic compromise in the first few days of life. In the brains of newborn and 1-d-old rats, carbohydrate and energy metabolism is much more resistant to compromise by anoxia than in 7-d-old rats (Duffy et al., 1975). These authors have also shown that the survival rate for perinatal rats under severe hypoxic conditions decreases dramatically with age. In the present experiments, we extended the hypobaric exposure time for 2-dold rats from 75 to $120 \mathrm{~min}$ and found that this both increased the severity of brain damage and decreased the survival rate. Thus, by more severely taxing the energy metabolic systems of the 2-d-old rat, we obtained a profile of sensitivity to hypoxia/ ischemia that correlated more closely with that to NMA toxicity. This suggests that NMA receptors are not insensitive to hypoxia/ ischemia on postnatal day 2 but that metabolic systems are less easily overwhelmed by hypoxic/ischemic conditions during that period. The paucity of glutamatergic terminals in early developmental stages (there are presumably less glutamatergic synapses formed on day 2 than on day 6) might also account for the relative resistance of the 2 -d-old rat brain to hypoxic/ischemic insults compared to older age groups. On the other hand, we observed that the neuronal response to either NMA or hypoxia/ischemia on day 2 was characterized by a more profound display of dendritic swelling and less pronounced degeneration of cell bodies than was seen on day 6 . This suggests that the NMA receptor system may have an immature pattern of distribution or may be subject to some other type of immaturity that modifies its potential for mediating excitotoxic phenomena at this age. Thus, it may be necessary to invoke both immaturity of NMA receptors and resistance of metabolic systems in the 2-d-old rat to explain the discrepancy noted above.
Also relevant to the interpretation of our findings is the relative insensitivity of the infant rat brain to the toxic action of kainic acid (Campochiaro and Coyle, 1978). It is thought that the first and second most prevalent types of excitatory amino acid (EAA) receptor in mammalian brain are NMA and kainic acid receptors, respectively. It is also believed that Glu acts at all subtypes of EAA receptors. If leakage of endogenous Glu from the intra- to extracellular compartment and excessive interaction of Glu with EAA receptors is the explanation for neuronal degeneration under hypoxic/ischemic conditions, and if, during certain stages of development, NMA receptors are extremely sensitive and kainic acid receptors relatively insensitive to EAA agonist interaction, it seems likely that endogenous Glu would exert its excitotoxic action preferentially through NMA receptors during that developmental period. This conclusion is further supported by the observation that MK-801, an NMA antagonist, powerfully protects all vulnerable neuronal populations in the infant rat brain against hypobaric/ischemic damage.

Observations suggesting hypersensitivity of NMA receptors during certain stages of development have been reported in electrophysiological studies. Responses of hippocampal pyramidal neurons to NMA are maximal in 5- to 9-d-old rats and decline progressively with age (Hamon and Heinemann, 1988). Visual responses of cortical neurons can be blocked much more effectively by NMA receptor antagonists in kittens than in adult cats (Tsumoto et al., 1987). NMA receptors reportedly may be critically involved in developmental plasticity (Cline et al., 1987; Cotman and Iversen, 1987; Kleinschmidt et al., 1987; Rauschecker and Hahn, 1987; Lincoln et al., 1988), including promotion of the formation of synaptic connections (Aruffo et al., 1987; Pearce et al., 1987; Balazs et al., 1988). Paradoxically, it appears that NMA receptors in early life may have the dual potential of promoting the development of CNS neurons or destroying such neurons, and it remains to be determined how these 2 properties interrelate.

It is noteworthy that significant intracerebral bleeding was observed in infant rats subjected to hypobaric/ischemic conditions in the 2-6 d period and that the bleeding was frequently associated with a focus of ischemic neuronal necrosis. While the mechanism of such bleeding is unclear, it may be significant that a similar bleeding pattern was noted in the brains of 2- to 6-d-old rats following injection of NMA into the head of the caudate nucleus and that the bleeding in these brains often occurred in the same regions where neurons were undergoing acute necrosis. Since the bleeding following either hypoxia/ischemia or NMA injection was associated with neurodegenerative lesions induced presumably by an excitotoxic mechanism, we postulate that there is something about an excitotoxic process in this early stage of development that promotes intracerebral bleeding. This is potentially an important point since much of the perinatal brain damage that occurs in the human, especially in premature infants, is accompanied by bleeding into the brain (Kovnar and Volpe, 1982). In addition, it should be noted that periventricular neurons lying immediately beneath the germinal matrix were particularly sensitive to injury during this early time interval. Interestingly, this midline periventricular layer is often the site of origin of intracerebral hemorrhage in premature human infants (Volpe, 1981; Larroche, 1984; Allan and Volpe, 1986).

It should be noted that the present study examines only the very acute cytopathological changes associated with hypoxia/ ischemia. The acute type of reaction shown here is identical in 
time course and appearance to that which occurs in the arcuate nucleus of the hypothalamus following subcutaneous administration of Glu to immature rodents or monkeys (Olney, 1971; Ikonomidou et al., 1989). When neurons show this fulminating type of response, consisting of massive perikaryal swelling and nuclear pyknosis, it is quite clear that a process of acute cell death is being witnessed; in fact, these cells are usually phagocytized and biologically degraded within the first 6-24 hr after the acute injury (Olney, 1971). It seems likely that many additional neurons go on to die by a more protracted degenerative process in the perinatal hypoxic/ischemic brain. This would be consistent with the findings of Choi et al. (1987) that cultured cerebrocortical neurons, after very brief exposure to abnormal Glu concentrations, go on to degenerate slowly by a calciumdependent process. It is possible that heightened sensitivity of the NMA receptor during development might render neurons particularly vulnerable to this slow type of degeneration following only minor degrees of hypoxia/ischemia. Since this type of subacute mechanism might contribute substantially to the ultimate severity of brain damage and disability associated with perinatal hypoxia/ischemia, it is clearly a subject requiring further research attention.

While we hypothesize, in general, that the developing rat brain may be subject to a 10 d period (days 4-14) of heightened NMA receptor sensitivity, it should be noted that each neuronal population displayed its own temporal pattern of heightened vulnerability. If the developing human CNS is similarly subject to a transitory period of heightened NMA receptor sensitivity, it is likely that it would span a period of months rather than days and that an ischemic insult would affect a different combination of neuronal populations, depending on which were closest to their period of peak sensitivity at the time of insult. Thus, it is conceivable that any of several developmental neuropsychopathology syndromes might result depending on the timing of the hypoxic/ischemic event. We conclude, therefore, that heightened sensitivity of the NMA receptor system during development may be an important mechanism to explore for potential clues to the etiopathogenesis of neuropsychiatric disorders.

\section{References}

Allan, W. C., and J. J. Volpe (1986) Periventricular-intraventricular hemorrhage. Pediatr. Clin. North Am. 33: 47-64.

Aruffo, C., R. Ferszi, A. G. Hildebrandt, and J. Ccrvos-Navarro (1987) Low doses of L-monosodium glutamate promote neuronal growth and differentiation in vitro. Dev. Neurosci. 9: 228-239.

Balazs, R., N. Hack, and O. S. Jorgensen (1988) Stimulation of the $\mathrm{N}$-methyl-D-aspartate receptor has a trophic effect on differentiating cerebellar granule cells. Neurosci. Lett. 87: 80-86.

Benveniste, H., J. Drejer, A. Schousboe, and N. H. Diemer (1984) Elevation of extracellular concentrations of glutamate and aspartate in rat hippocampus during transient cerebral ischemia monitored by intracerebral microdialysis. J. Neurochem. 43: 1369-1374.

Boast, C. A., S. C. Gerhardt, G. Pastor, J. Lehmann, P. E. Etienne, and J. M. Liebman (1988) The NMDA antagonists CGS 19755 and CPP reduce ischemic brain damage in gerbils. Brain Res. 442: 345-348.

Campochiaro, P., and J. T. Coyle (1978) Ontogenetic development of kainate neurotoxicity: Correlates with glutamatergic innervation. Proc. Natl. Acad. Sci. USA 75: 2025-2029.

Choi, D. W., M. Maulucci-Gedde, and A. R. Kriegstein (1987) Glutamate neurotoxicity in cortical cell culture. J. Neurosci. 7:357-368.

Clark, G. D., and S. M. Rothman (1987) Blockade of excitatory amino acid receptors protects anoxic hippocampal slices. Neuroscience 21 : 665-671.

Cline, H. T., E. A. Debski, and M. Constantine-Paton (1987) N-methyl-D-aspartate antagonist desegregates eye-specific stripes. Proc. Natl. Acad. Sci. USA 84: 4342-4345.
Cotman, C. W., and L. L. Iversen (1987) Excitatory amino acids in the brain - focus on NMDA receptors. TINS 10: 263-265.

Drejer, J., H. Benveniste, N. H. Diemer, and A. Schousboe (1985) Cellular origin of ischemia-induced glutamate release from brain tissue in vivo and in vitro. J. Neurochem. 45: 145-151.

Duffy, T. E., S. J. Kohle, and R. C. Vannucci (1975) Carbohydrate and energy metabolism in perinatal rat brain: Relation to survival in anoxia. J. Neurochem. 24: 271-276.

Gill, R., A. C. Foster, and G. N. Woodruff (1987) Systemic administration of MK-801 protects against ischemia-induced hippocampal neurodegeneration in the gerbil. J. Neurosci. 7: 3343-3349.

Goldberg, M. P., J. H. Weiss, P.-C. Pham, and D. W. Choi (1987) $\mathrm{N}$-methyl-D-aspartate receptors mediate hypoxic neuronal injury in cortical culture. J. Pharmacol. Exp. Ther. 243: 784-791.

Hagberg, H., P. Andersson, I. Kjellmer, K. Thiringer, and M. Thordstein (1987) Extracellular overflow of glutamate, aspartate, GABA and taurine in the cortex and basal ganglia of fetal lambs during hypoxiaischemia. Neurosci. Lett. 78: 311-317.

Hamon, B., and U. Heinemann (1988) Developmental changes in neuronal sensitivity to excitatory amino acids in area CAl of the rat hippocampus. Dev. Brain Res. 38: 286-290.

Ikonomidou, C., G. Frierdrich, K. Shahid Salles, J. Labruyere, M. Price, and J. W. Olney (1988) Glutamate-like damage in infant rat brain produced by hypobaric-ischemic conditions. In Frontiers in Excitatory Amino Acid Research, J. Lehmann, E. A. Cavalheiro, and L. Turski, eds., pp. 657-660, Liss, New York.

Ikonomidou, C., M. T. Price, J. L. Mosinger, G. Frierdrich, J. Labruyere, K. Shahid Salles, and J. W. Olney (1989) Hypobaric-ischemic conditions produce glutamate-like cytopathology in infant rat brain. J. Neurosci. 9: 1693-1700

Kleinschmidt, A., M. F. Bear, and W. Singer (1987) Blockade of "NMDA" receptors disrupts experience dependent plasticity of kitten striate cortex. Science 238: 355-358.

Kochhar, A., J. A. Zivin, P. D. Lyden, and V. Mazzarella (1988) Glutamate antagonist therapy reduces neurologic deficits produced by focal central nervous system ischemia. Arch. Neurol. 45: 148-154.

Koenig, J. F. R., and R. A. Klippel (1967) The Rat Brain. A Stereotaxic Atlas of the Forebrain and Lower Parts of the Brain Stem, Robert and Krieger, Huntington, NY.

Kovnar, E., and J. J. Volpe (1982) Current concepts in neonatal neurology: Hypoxic-ischemic brain injury and intraventricular hemorrhage. Perinatol. Neonatol. 6: 51-63, 81-92.

Larroche, J.-C. (1984) Perinatal brain damage. In Greenfield's $\mathrm{Neu}$ ropathology, J. H. Adams, J. A. N. Corsellis, and L. W. Duchen, eds., pp. 451-489, Edward Arnold, London.

Lawrence, J. J., T. A. Fuller, and J. W. Olney (1987) MK-801 and PCP protect against ischemic neuronal degeneration in the gerbil hippocampus. Soc. Neurosci. Abstr. 13: 1079.

Lincoln, J., R. Coopersmith, E. W. Harris, C. W. Cotman, and M. Leon (1988) NMA receptor activation and early olfactory learning. Dev. Brain Res. 39: 309-312.

McDonald, J. W., F. S. Silverstein, and M. V. Johnston (1987) MK801 protects the neonatal brain from hypoxic/ischemic damage. Eur. J. Pharmacol. 140: 359-361.

McDonald, J. W., F. S. Silverstein, and M V. Johnston (1988) Neurotoxicity of N-methyl-D-aspartate is markedly enhanced in developing rat central nervous system. Brain Res. 459: 200-203.

Olney, J. M., G. E. Gray, G. Frierdrich, K. Shahid Salles, J. Labruyere, M. T. Price, and J. W. Olney (1987) Hypoxia-ischemia causes glutamate-like neuropathological changes in infant rat brain. Soc. Neurosci. Abstr. 13: 1553.

Olney, J. W. (1971) Glutamate-induced neuronal necrosis in the infant mouse hypothalamus. J. Neuropathol. Exp. Neurol. 1: 75-90.

Olney, J. W., C. Ikonomidou, J. Mosinger, K. Shahid Salles, and G. Frierdrich (1988a) Role of the N-methyl-D-aspartate receptor in devclopmental psychoncuropathology. Soc. Ncurosci. Abstr. 14:417.

Olney, J. W., M. T. Price, J. Labruyere, M. Mueller, and E. Silverman (1988b) Comparative efficacy of thiobarbiturates and other agents in preventing glutamate-induced or ischemic neuronal degeneration in chick retina. Neurosci. Lett. (in press).

Olney, J. W., C. Ikonomidou, J. L. Mosinger, and G. Frierdrich (1989) MK-801 prevents hypobaric-ischemic neuronal degeneration in infant rat brain. J. Neurosci. 9: 1701-1704.

Park, C., D. G. Nehls, E. Ozyurt, D. I. Graham, and J. McCulloch (1987) Ischemic brain damage is reduced by systemic administration 
of the N-methyl-D-aspartate (NMDA) antagonist, MK-801. Soc. Neurosci. Abstr. 13: 1029.

Pearce, I. A., M. A. Cambray-Deakin, and R. D. Burgoyne (1987) Glutamate acting on NMDA receptors stimulates neurite outgrowth from cerebellar granule cells. FEBS Lett. 223: 143-147.

Prince, D. A., and H. R. Freeser (1988) Dextromethorphan protects against cerebral infarction in a rat model of hypoxia-ischemia. Neurosci. Lett. 85: 291-296.

Rauschecker, J. P., and S. Hahn (1987) Ketamine-xylazine anaesthesia blocks consolidation of ocular dominance changes in kitten visual cortex. Nature 326: 183-185.

Rothman, S. M. (1984) Synaptic release of excitatory amino acid neurotransmitter mediates anoxic neuronal death. J. Neurosci. 4:1884 1891.

Rothman, S. M., J. H. Thurston, and R. E. Hauhart (1987) Delayed neurotoxicity of excitatory amino acids in vitro. Neuroscience 22 : $471-480$.

Sherwood, N M., and P. S. Timiras (1970) A Stereotaxic Atlas of the Developing Rat Brain, University of California Press, Berkeley.

Silverstein, F. S., K. Buchanan, and M. V. Johnston (1986) Perinatal hypoxia-ischemia disrupts striatal high affinity $\left({ }^{3} \mathrm{H}\right)$ glutamate uptake into synaptosomes. J. Neurochem. 47: 1614-1619.

Simon, R. P., J. H. Swan, T. Griffiths, and B. S. Meldrum (1984) Blockade of $\mathrm{N}$-methyl-D-aspartate receptors may protect against ischemic damage in the brain. Science 226: 850-853.
Steinberg, G. K., J. Saleh, and D. Kunis (1988) Delayed treatment with dextromethorphan and dextrorphan reduces cerebral damage after transient focal ischemia. Neurosci. Lett. 89: 193-197.

Tsumoto, T., K. Hagihara, H. Sato, and Y. Hata (1987) NMA receptors in the visual cortex of young kittens are more effective than those of adult cats. Nature 327: 513-514.

Volpe, J. J. (1981) Neonatal intraventricular hemorrhage. N. Engl. J. Med. 304: 886-891.

Watkins, J. C., and H. J. Olverman (1987) Agonists and antagonists for excitatory amino acid receptors. TINS 10: 265-272.

Weiss, J., M. P Goldberg, and D. W. Choi (1986) Ketamine protects cultured neocortical neurons from hypoxic injury. Brain Res. 380: $186-190$.

Winer, B. J. (1971) Statistical Principles in Experimental Design, McGraw-Hill, New York.

Wolf, G., and G. Keilhoff (1984) Kainate and glutamate neurotoxicity in dependence on postnatal development with special reference to hippocampal neurons. Dev. Brain Res. 14: 15-21.

Wong, E. H. F., J. A. Kemp, T. Priestley, A. R. Knight, G. N. Woodruff, and L. L. Iverson (1986) The anticonvulsant MK-801 is a potent N-methyl-D-aspartate antagonist. Proc. Natl. Acad. Sci. USA 83. 7104-7108 